\title{
Noise Exposure From High-Flow Nasal Cannula Oxygen Therapy: A Bench Study on Noise Reduction
}

\author{
Takamitsu Kubo CE, Hiroaki Nakajima CE, Ryo Shimoda CE, Tatsuya Seo CE, Yurie Kanno CE, \\ Toshikazu Kondo CE, and Sunao Tamai MD PhD
}

\begin{abstract}
BACKGROUND: High-flow nasal cannula (HFNC) oxygen therapy produces noise at a level such that patients often complain. However, the noise level has not been measured digitally. METHODS: We evaluated 3 types of HFNCs without filters and 2 types with filters attached for noise reduction. Optiflow (with and without a filter), MaxVenturi (with and without a filter) and AIRVO2 (without a filter only) were positioned at the center of a hospital room. We measured the noise levels at the distance of $1 \mathrm{~m}$ from the equipment at various total flows $(30,40,50,60 \mathrm{~L} / \mathrm{min})$ and $\mathrm{F}_{\mathrm{IO}_{2}}(0.40,0.60$, and 0.90$)$. RESULTS: Noise levels were increased with the AIRVO2 and MaxVenturi when total flow and $\mathrm{F}_{\mathrm{IO}_{2}}$ were increased. Noise levels decreased with the MaxVenturi when a filter was used. The noise level did not change with the Optiflow when total flow and $\mathrm{F}_{\mathrm{IO}_{2}}$ were increased. The noise level decreased in the groups with AIRVO2 and Optiflow compared with MaxVenturi without a filter. CONCLUSIONS: The findings in this study show that the noise level of HFNC/Venturi could be reduced by attaching an intake filter. However, the noise level of HFNC/blender and HFNC/turbine decreased in comparison with HFNC/Venturi without an intake filter. Key words: high flow nasal cannula; optiflow; maxventuri; airvo2; noise; noise level; hfnc. [Respir Care 2018;63(3):267-273. (C) 2018 Daedalus Enterprises]
\end{abstract}

\section{Introduction}

Environmental noise levels in hospitals have increased steadily over the past 50 years. It has been reported that average daytime hospital noise levels have increased from $57 \mathrm{~dB}$ to $72 \mathrm{~dB} .{ }^{1}$ Noise may cause such adverse effects as cardiovascular disturbances, communication errors among patients and staff, longer hospital stays, unsteady sleep patterns, and increased annoyance. ${ }^{2}$ Furthermore, health care providers exposed to elevated noise levels may be at risk for hearing damage and unintended distractions that may lead to increased cognitive errors. ${ }^{3}$

Mr Kubo, Mr Nakajima, Mr Shimoda, Mr Seo, Ms Kanno, and Mr Kondo are affiliated with the Medical Equipment Center, Shizuoka Cancer Center Hospital, Shizuoka Prefecture, Japan. Dr Tamai is affiliated with the Division of Anesthesiology, Shizuoka Cancer Center Hospital, Shizuoka Prefecture, Japan.

The authors have disclosed no conflicts of interests.

Correspondence: Takamitsu Kubo, Clinical Engineer Shizuoka Cancer Center, 1007 Shimonagakubo, Nagaizumi-cho, Sunto-gun, Shizuoka Prefecture, 4118777 Japan. E-mail: t.kubo@scchr.jp.

DOI: $10.4187 /$ respcare. 05668
High-flow nasal cannula (HFNC) oxygen therapy can improve oxygenation by delivering humidified oxygen and which may reduce the need for mechanical ventilation through dead space washout of the upper airway. Three types of HFNC systems are available clinically: the Venturi (HFNC/Venturi), the air-oxygen blender (HFNC/blender), and the turbine (HFNC/turbine). The HFNC/Venturi is typically applied when a medical gas outlet is inaccessible (eg, in recovery after minimally invasive surgery and during physical therapy or transfers). Patients often complain that the noise during HFNC oxygen therapy is uncomfortable. Becase the noise level has not been measured during HFNC oxygen therapy, we aimed to digitize the noise level of each type of HFNC system and determine the effect of attaching a respiratory filter on the noise level.

\section{Methods}

\section{Location}

Each type of HFNC system was located at the center of a hospital room $(4.2 \mathrm{~m} \times 3.4 \mathrm{~m} \times 2.7 \mathrm{~m})$ in the Shizuoka Cancer Center (Shinzuoka Prefecture, Japan). No patientwas present. The noise level was measured with the digitized 
sound pressure measuring instrument at a distance of $1 \mathrm{~m}$ from each HFNC system.

\section{See the Related Editorial on Page 371}

\section{Devices}

The components of the HFNC/Venturi included an MR850 heated humidifier (Fisher \& Paykel, Auckland, New Zealand), a MaxVenturi combination air/oxygen entrainment device (Maxtec, Salt Lake City, Utah), an oxygen analyzer with flow meter, and an RT202 single-limb heated-wire circuit with a medium-sized adult cannula (Fisher \& Paykel). An oxygen tank was used as the driving source (Fig. 1).

The components of the HFNC/blender were an MR850 heated humidifier, an Optiflow, and an RT202 single-limb heating-wire circuit with a medium-sized adult cannula (Fisher \& Paykel). Medical gas outlets of air and oxygen were used as the driving source (Fig. 2).

The components of the HFNC/turbine were the AIRVO2, an oxygen flow meter, a 900PT501 tube, and a chamber kit with a medium-sized adult cannula (Fisher \& Paykel) attached. We used an electric power supply outlet as the driving source (Fig. 3).

The Optiflow set-up included a respiratory filter for preventing cross-contamination (350/5865Z, Covidien, Mansfield, MA), with a dead-space volume of $99 \mathrm{~mL}$ and flow resistances of $0.6 \mathrm{~cm} \mathrm{H}_{2} \mathrm{O}$ at $30 \mathrm{~L} / \mathrm{min}$ and $1.5 \mathrm{~cm} \mathrm{H}_{2} \mathrm{O}$ at $60 \mathrm{~L} / \mathrm{min}$, which was applied to the air intake as a noise reduction. The MaxVenturi set-up included a respiratory filter for preventing cross-contamination (FH603003, Ventlab, Grand Rapids, MI), with a dead-space volume of $54 \mathrm{~mL}$ and flow resistances of $<1 \mathrm{~cm} \mathrm{H}_{2} \mathrm{O}$ at $30 \mathrm{~L} / \mathrm{min}$ and $<2 \mathrm{~cm} \mathrm{H}_{2} \mathrm{O}$ at $60 \mathrm{~L} / \mathrm{min}$, which was applied to the air intake as a noise reduction (Fig. 1, Fig. 4).

All noise levels were measured with a SL-1320 digital sound meter with a 0.5 -inch $(12.7 \mathrm{~mm})$ electric condenser microphone (Custom Corp, Tokyo, Japan). The device includes a windscreen to minimize artifacts and has a measurement range of $40-130 \mathrm{~dB}$, with $0.1-\mathrm{dB}$ resolution and an accuracy of $\pm 2 \mathrm{~dB}$. A 1-s refresh rate was used.

\section{Protocol}

Before each study began, the noise level in the hospital room with the bedside monitor turned off was measured as a control. First, as shown in Figures 1-3, we positioned each type of HFNC so that it hung freely. Second, we measured the noise levels for each type of

\section{QUICK LOOK}

\section{Current knowledge}

High levels of ambient noise are associated with sleep disturbance, the risk of delirium, and exacerbation of cognitive dysfunction. All clinically available highflow nasal cannula (HFNC) systems produce constant noise. However, these noise levels have not been digitally measured, and it remains unclear whether a standard respiratory filter could alter the intensity of this sound.

\section{What this paper contributes to our knowledge}

There were no significant differences in the noise levels of the HFNC/blender with use of a filter. In contrast, the HFNC/Venturi without a filter was the loudest, but the noise level was reduced by attaching a filter at the air intake. The lowest noise produced between the 3 systems was with the $\mathrm{HFNC} /$ turbine using an $\mathrm{F}_{\mathrm{IO}_{2}}<0.60$.

HFNC 5 times with 30 -s intervals until the value became stable at a distance of $1 \mathrm{~m}$ with the following settings: total flows of 30, 40, 50, and $60 \mathrm{~L} / \mathrm{min} ; \mathrm{F}_{\mathrm{IO}_{2}}$ values of $0.40,0.60$, and 0.90 ; without a filter; with a filter attached to the air intake side of the HFNC/Venturi; and with a filter attached to the total flow outtake side of the HFNC/blender. We also measured noise levels 10 times in the same manner as above with the HFNC/turbine without the filter.

\section{Statistical Analysis}

All the data are expressed as mean $\pm \mathrm{SD}$. The comparisons among the measured noise levels with the HFNC/Venturi, the HFNC/blender, and the HFNC/turbine, with and without the filters, were analyzed with the Wilcoxon rank-sum test (for unmatched pairs) and the Wilcoxon signed-rank test (for matched pairs). $P$ values $<.05$ were considered statistically significant. Calculations were performed with a statistical package (add-in software for Microsoft Excel 2010, Social Survey Research Information, Japan).

\section{Results}

For the HFNC/blender (Optiflow), the noise levels in the hospital room before the study began were $42.7 \pm 1.34 \mathrm{~dB}$, and there were no significant changes even when total flow increased $(P=.07)$. Increasing $\mathrm{F}_{\mathrm{IO}_{2}}$ also did not significantly affect noise levels $(P=.31)$. 


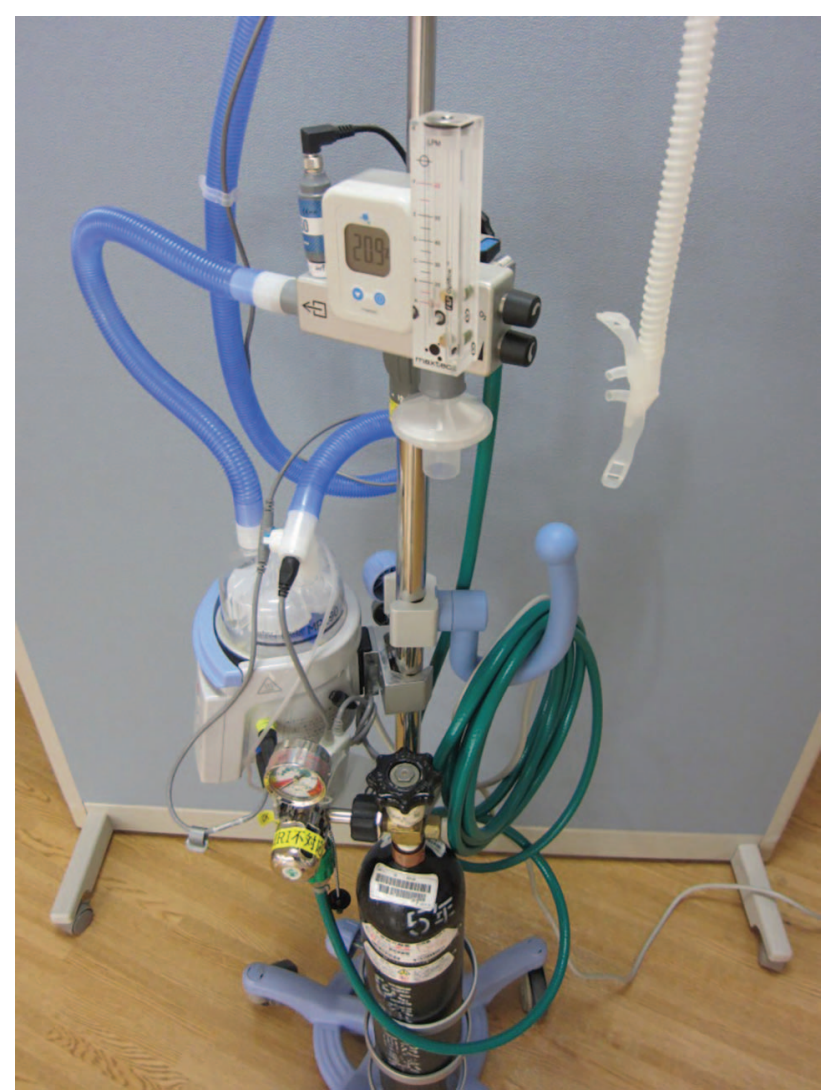

Fig. 1. Experimental setup for MaxVenturi. An exclusive circuit and a filter for noise reduction were attached on the air intake side of MaxVenturi using the oxygen cylinder, which is the driving source. The tip of the nasal cannula hung freely.

Inaddition, the difference in noise levels when using a filter was not significant when the total flow was $50 \mathrm{~L} / \mathrm{min}$ with $\mathrm{F}_{\mathrm{IO}_{2}}=0.60$, which theoretically might have been useful $(P=.068)$. However, the noise level of this device was significantly lower than that of the HFNC/Venturi without a filter $(P=.03$; Fig. 5).

For the HFNC/Venturi (MaxVenturi), the noise levels in our hospital room before the study began were $42.1 \pm 1.21 \mathrm{~dB}$. The noise level increased significantly when total flow and $\mathrm{F}_{\mathrm{IO}_{2}}$ increased $(P=.03)$. The noise level was significantly reduced with the filter compared with no filter $(P=.03$; Fig. 6$)$.

For the HFNC/turbine (AIRVO2), the noise levels in the hospital room before the study began were $42.6 \pm 0.95 \mathrm{~dB}$. The noise level, which increased in proportion to increases in $\mathrm{F}_{\mathrm{IO}_{2}}$ and total flow $(P=.005)$, was significantly lower than the noise level of the HFNC/Venturi without the filter $(P=.01)$. The noise level of the HFNC/turbine was significantly lower than the HFNC/Venturi with the filter, except when $\mathrm{F}_{\mathrm{IO}_{2}}=0.90$ (Fig. 7).

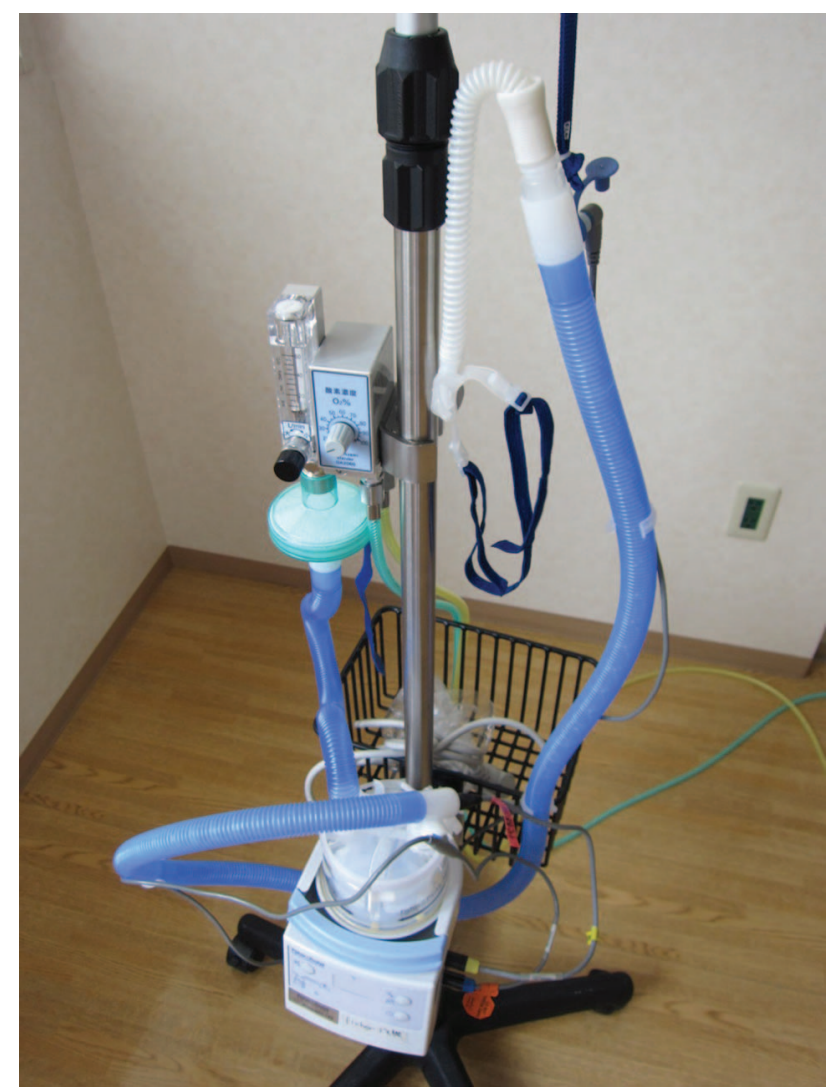

Fig. 2. Experimental setup for Optiflow. An exclusive circuit and a filter for noise reduction were attached on the total flow outtake side of Optiflow using the medical gas outlet for oxygen and air as the driving source. The tip of the nasal cannula hung freely.

\section{Discussion}

Excessive noise is an evident and ubiquitous problem in hospitals. Noise can impair both physiologic and psychologic homeostasis of patients and staff. Various types of oxygen therapy, including HFNC, are sources of daily noise. HFNCs, which produce high gas velocity, can be divided into 3 groups according to flow methods: gas blender using medical gas as a driving source, Venturi-effect through entrainment of ambient air using medical gas, and turbine by entrainment of ambient air using an electric turbine system. We recommend the HFNC/Venturi in ambulatory patients who may leave the hospital room to walk during in-patient rehabilitation or transfers, because the HFNC/Venturi can be driven with an oxygen tank alone, without using electric power. We recommend the HFNC/Venturi or the HFNC/turbine when an air outlet is not installed in the hospital room. For patients with chronic 


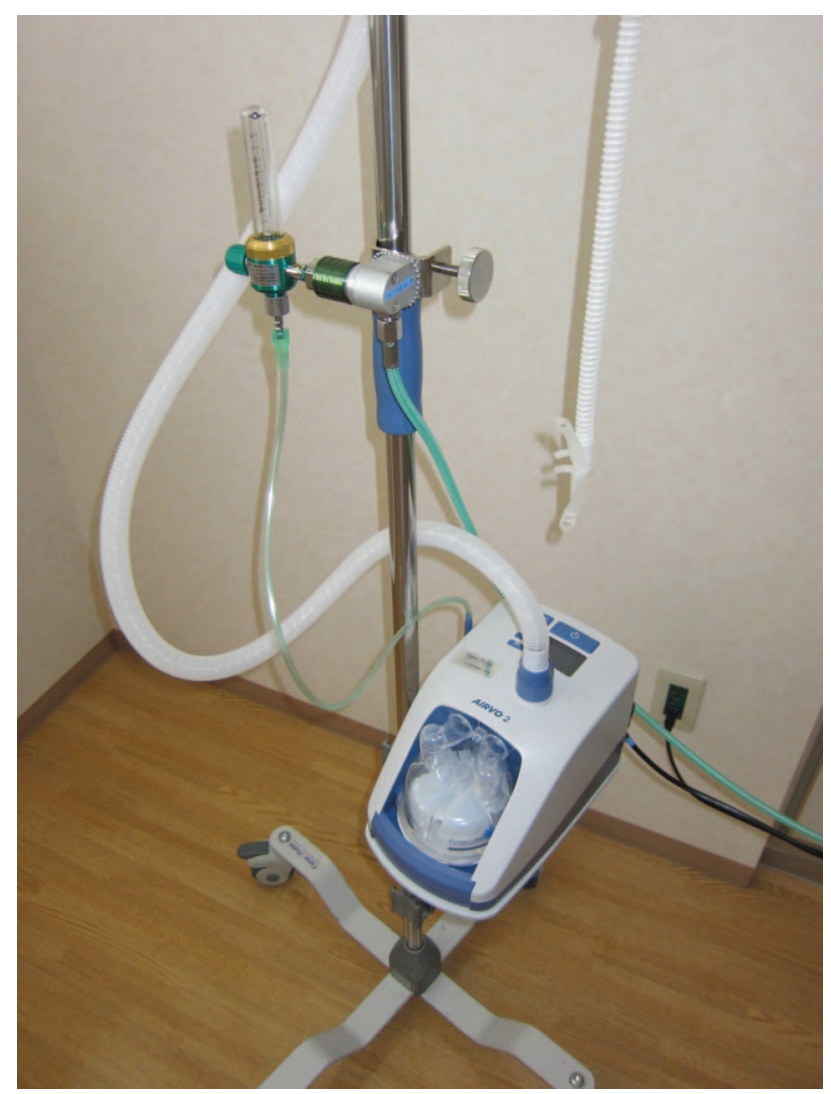

Fig. 3. Experimental setup for AIRVO2. An oxygen flow meter and an exclusive circuit were attached to AIRVO2 using a power supply outlet (consumption of electric current was $2.4 \mathrm{~A}$ ). The tip of the nasal cannula hung freely.

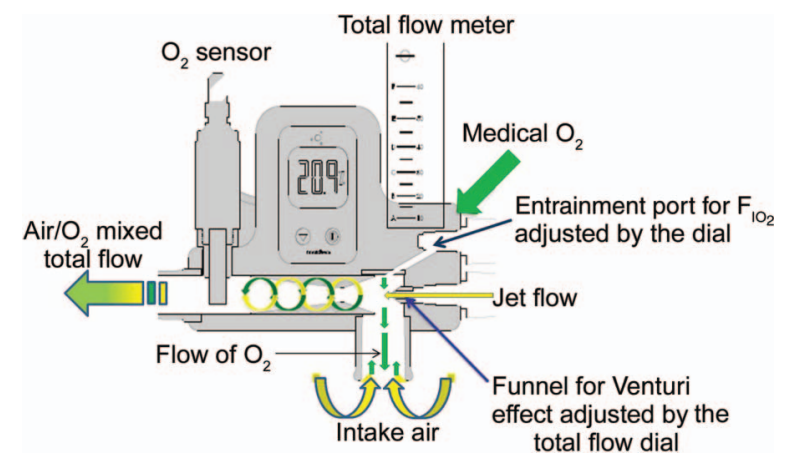

Fig. 4. Interior construction of MaxVenturi.

hypercapnic respiratory failure, we recommend the HFNC/turbine or the HFNC/blender, which can be set at an $\mathrm{F}_{\mathrm{IO}_{2}} \geq 0.21$. Additionally, we often need an $\mathrm{F}_{\mathrm{IO}_{2}}$ of 1.0 for patients with terminal cancer. Therefore, the HFNC/turbine cannot be used, as the high concentration oxygen alarm will continuously go off when the $\mathrm{F}_{\mathrm{IO}_{2}}$ is set $>0.96$. There are various reasons for choosing a device, choosing one that can reduce noise levels for patients and can support comfortable hospital environments should be an important factor.

\section{HFNC/Venturi}

Conventional humidified oxygen therapy (eg, T-piece) produces total flow according to the Venturi-Bernoulli principle, creating a narrow orifice to increase velocity and to regulate $\mathrm{F}_{\mathrm{IO}_{2}}$. As $\mathrm{F}_{\mathrm{IO}_{2}}$ increases, the entrainment port becomes smaller, and thus total flow decreases. Therefore, it seems logical that noise would decrease in proportion to $\mathrm{F}_{\mathrm{IO}_{2}}$. Berg et $\mathrm{al}^{4}$ reported that noise can be reduced from $70 \mathrm{~dB}$ to $55 \mathrm{~dB}$ using a modified T-piece.

The HFNC/Venturi used in our study has a knob to adjust $\mathrm{F}_{\mathrm{IO}_{2}}$ and another to adjust total flow. Both are used to maintain stable total flow by adjusting air-entrainment and the Venturi effect (Fig. 4). When we increased $\mathrm{F}_{\mathrm{IO}_{2}}$, air intake decreased, which resulted in decreased total flow. We confirmed this reduction with the flow meter. Then, to increase total flow, we adjusted the dial for the Venturi effect to compensate for the loss of flow from reduced air entrainment.

We believe this mechanism was the factor that increased noise levels in proportion to $\mathrm{F}_{\mathrm{IO}_{2}}$. A respiratory filter applied to reduce the noise in this study was found to be effective. We also highly recommend attaching a filter to the intake side, because research has shown that fine particles and bacteria are present in room air within a respiratory care center. ${ }^{5}$ In our study, the filter became considerably dirty in only 2 weeks.

\section{HFNC/Blender}

In contrast, the HFNC/blender produces total flow from mixing oxygen and air in a mixing chamber, using medical gas pressure as the driving source. The noise level is dependent on total flow and not on $\mathrm{F}_{\mathrm{IO}_{2}}$. However, we hypothesized that the noise level was lower because it did not involve the Venturi effect, which takes in a large quantity of air. Moreover, the noise level did not change when we attached a filter. Therefore, it is not necessary to attach a filter to the flow outlet on this device because it does not reduce noise and can increase flow resistance. We found that the noise level of the HFNC/blender was less than or equal to that of the HFNC/Venturi with a filter when the total flow was $30 \mathrm{~L} / \mathrm{min}$ and $40 \mathrm{~L} / \mathrm{min}$.

\section{HFNC/Turbine}

The HFNC/turbine produces total flow by the intake of ambient air with a turbine driven by electric power. 


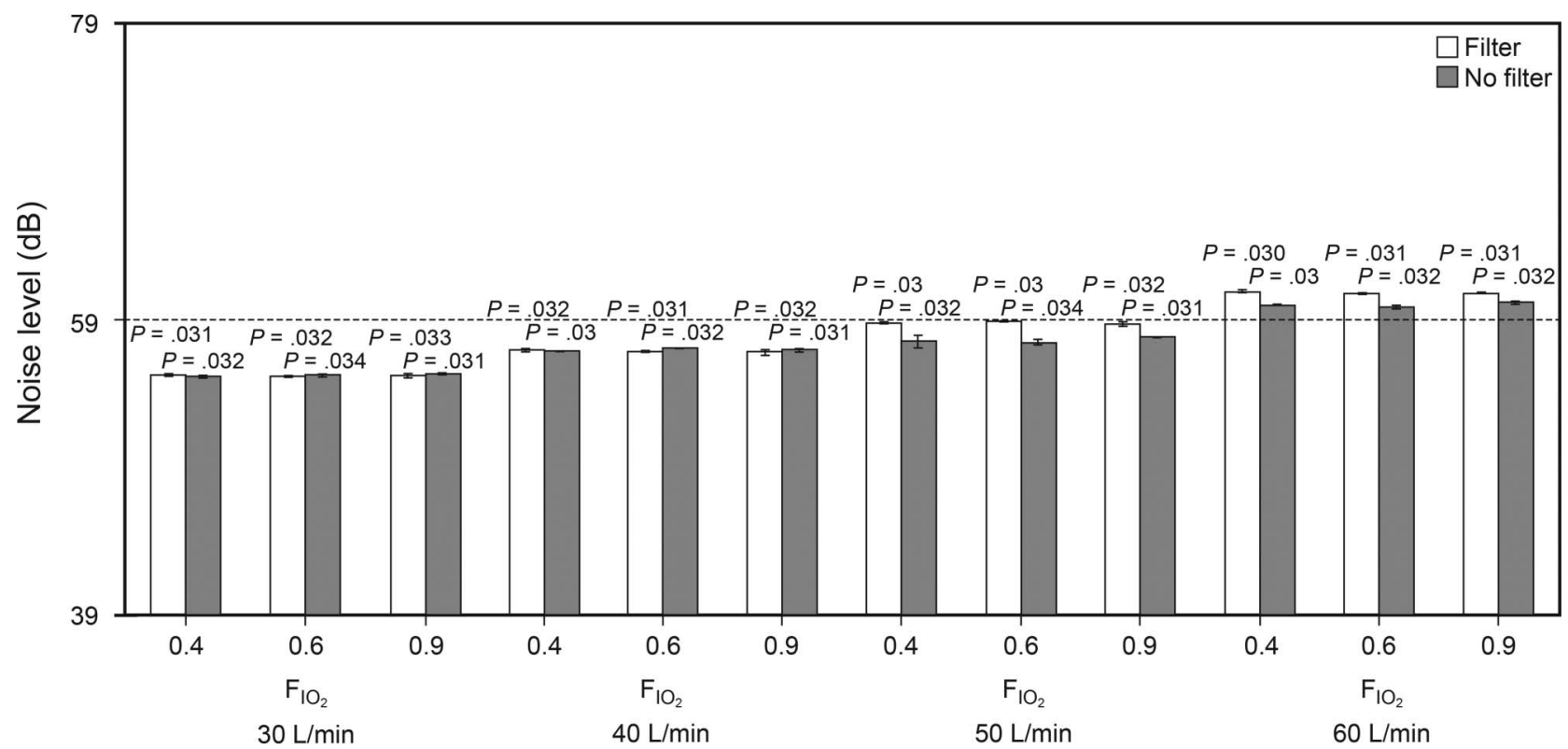

Fig. 5. Noise levels with and without a filter for the total flow and the oxygen concentration, respectively, on the HFNC/blender (Optiflow). All $P$ values vs. the HFNC/Venturi without a filter (see Fig. 6). Data are mean \pm SD.

When high levels of oxygen are required, increasing the oxygen flow is necessary because $\mathrm{F}_{\mathrm{IO}_{2}}$ decreases in proportion to the total flow. Therefore, the noise level in- tensified in proportion to $\mathrm{F}_{\mathrm{IO}_{2}}$ and total flow. However, compared with the HFNC/Venturi, which has the samemechanism of ambient air intake, the noise level was

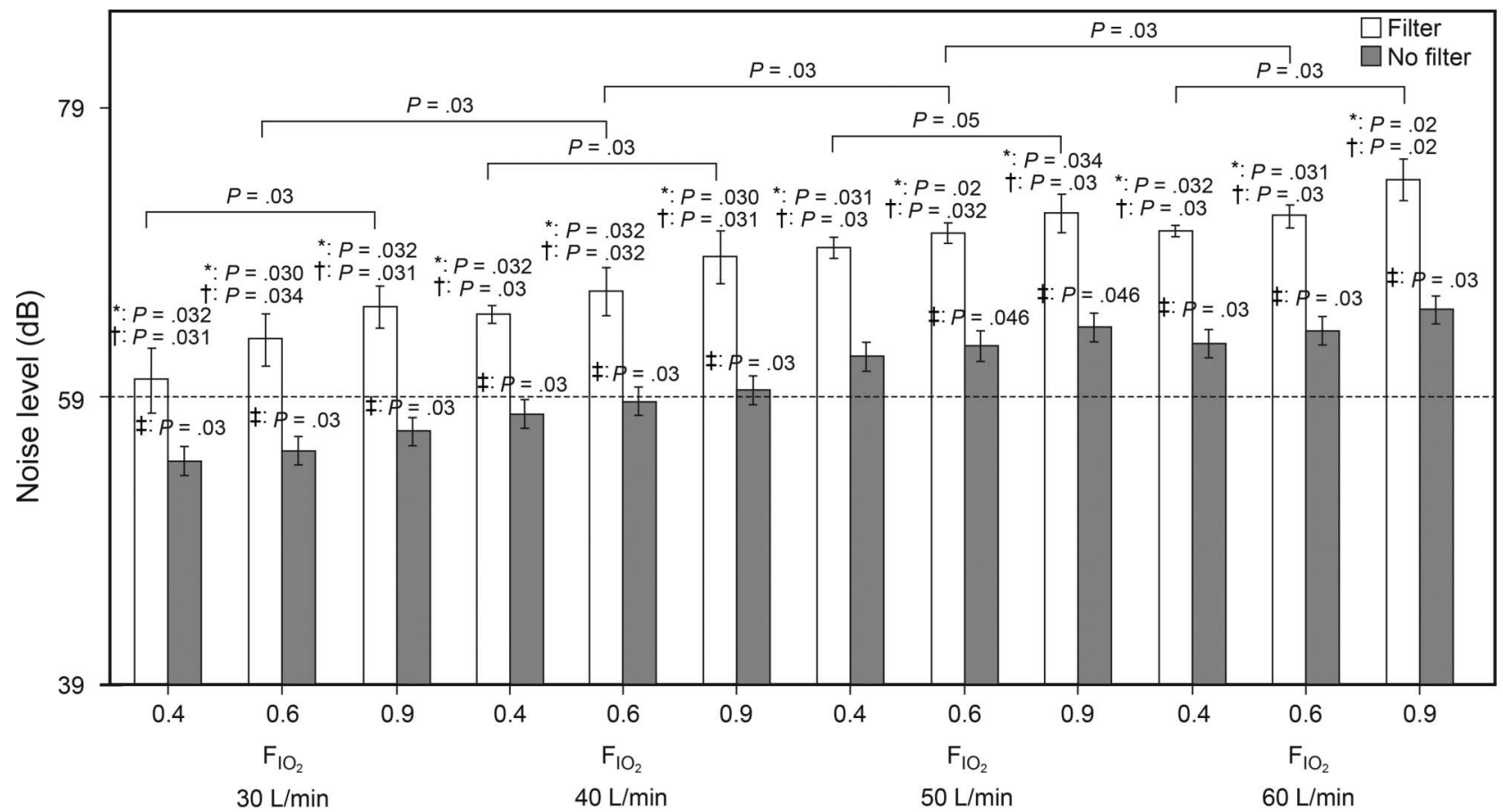

Fig. 6. Noise levels with and without a filter for the total flow and the oxygen concentration, respectively, on the HFNC/Venturi (MaxVenturi). * vs with a filter, $\dagger$ vs the $\mathrm{HFNC/blender} \mathrm{with} \mathrm{and} \mathrm{without} \mathrm{a} \mathrm{filter} \mathrm{(see} \mathrm{Fig.} \mathrm{5),} \ddagger$ vs. $\mathrm{F}_{1 \mathrm{O}_{2}}=0.40$ when the total flow was $50 \mathrm{~L} / \mathrm{min}$. Data are mean \pm SD. 


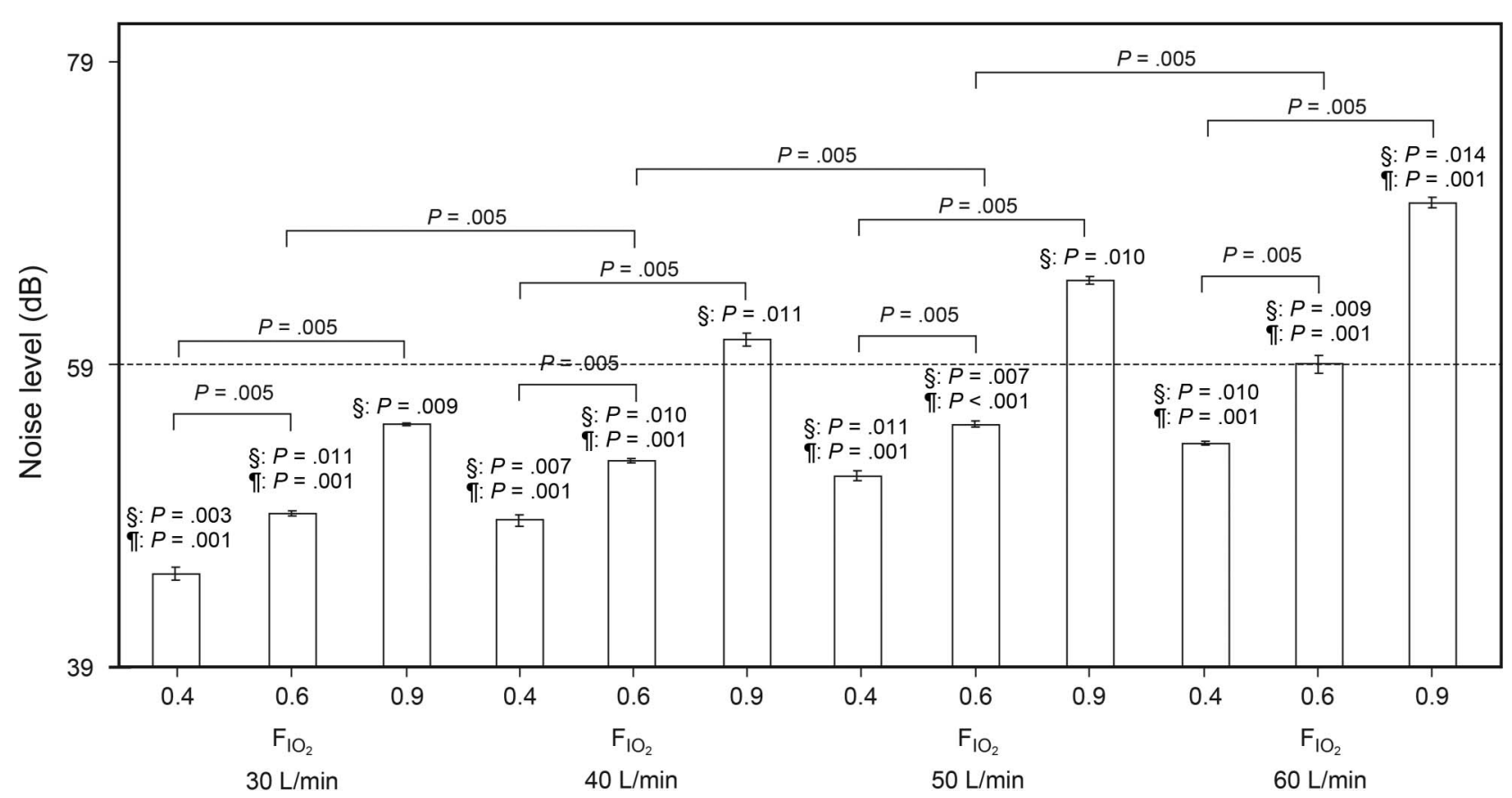

Fig. 7. Noise levels without a filter for the total flow and the oxygen concentration, respectively, on the HFNC/turbine (AIRVO2). $\S$ vs. the HFNC/Venturi without a filter (see Fig. 6). I vs. the HFNC/Nenturi with a filter. Data are mean \pm SD.

significantly lower because of design features that reduce sound, including several silicone-based dampers supporting the turbine.

\section{Cutoff Points of the Total Flow and $\mathrm{F}_{\mathrm{IO}_{2}}$}

Our study was based on noise levels of $59 \mathrm{~dB}$ for nighttime and $63 \mathrm{~dB}$ for daytime, which are noise levels reported to prevent sleep. ${ }^{6}$ For daytime use (ie, $63 \mathrm{~dB}$ ), total flow and $\mathrm{F}_{\mathrm{IO}_{2}}$ settings with the $\mathrm{HFNC/blender} \mathrm{can} \mathrm{be}$ unrestricted. However, with the HFNC/Venturi with a filter attached, total flow should be maintained at $\leq 40 \mathrm{~L} / \mathrm{min}$. Without a filter, noise exposure was quite significant with the HFNC/Venturi, so we recommend avoiding this device when possible. With the HFNC/turbine, total flow should be maintained $\leq 50 \mathrm{~L} / \mathrm{min}$ with $\mathrm{F}_{\mathrm{IO}_{2}} \leq 0.60$. For nighttime use (ie, $59 \mathrm{~dB}$ ), we recommend using the HFNC/turbine with $\mathrm{F}_{\mathrm{IO}_{2}} \leq 0.60$ (no adjustments required for the total flow), and we recommend avoiding the HFNC/blender and HFNC/Venturi as much as possible.

\section{Future Research}

A limitation of our study is that we did not obtain any data with human subjects, so we cannot assess subjective responses and opinions related to these noise levels.
People react to sound in different ways, and it may depend on whether they accept the noise levels as something necessary. Therefore, research involving human subjects is one of our future goals.

\section{Conclusions}

The noise levels of the HFNC/Venturi (Max Venturi) and the HFNC/turbine (AIRVO2) increased in proportion to increases in the $\mathrm{F}_{\mathrm{IO}_{2}}$ or the total flow. The noise level of the HFNC/blender (Optiflow) did not change significantly in relation to increases in the $\mathrm{F}_{\mathrm{IO}_{2}}$ or the total flow. The noise level of the HFNC/Venturi could be reduced by an intake filter, whereas the noise level of the HFNC/blender did not change significantly by attaching a filter. The noise levels of the HFNC/blender and the HFNC/turbine were lower than that of the HFNC/Venturi without a filter.

\section{REFERENCES}

1. Busch-Vishniac IJ, West JE, Barnhill C, Hunter T, Orellana D, Chivukula R. Noise levels in Johns Hopkins Hospital. J Acoust Soc Am 2005;118(6):3629-3645.

2. Choiniere DB. The effects of hospital noise. Nurs Adm Q 2010;34(4): 327-333. 


\section{Reducing NoIse From HFNC Systems}

3. Beyea SC. Noise: a distraction, interruption, and safety hazard. AORN J 2007;86(2):281-285.

4. Berg PW, Stuttmann R, Doehn M. [Noise in intensive care units. Noise reduction by modification of gas humidification] Anaesthesist 1997;46(10):856-859.

5. Chung FF, Lin HL, Liu HE, Lien AS, Hsiao HF, Chou LT, et al. Aerosol distribution during open suctioning and long-term surveil- lance of air quality in a respiratory care center within a medical center. Respir Care 2015;60(1):30-37.

6. Elbaz M, Leger D, Sauvet F, Champigneulle B, Rio S, Strauss M, et al. Sound level intensity severely disrupts sleep in ventilated ICU patients throughout a 24-h period: a preliminary 24-h study of sleep stages and associated sound levels. Ann Intensive Care 2017;7(1):25.

This article is approved for Continuing Respiratory Care Education credit. For information and to obtain your CRCE

(free to AARC members) visit 